\title{
Causal Analysis of Parameterized Atomic HAADF-STEM Across a Doped Ferroelectric Phase Boundary
}

Christopher Nelson ${ }^{1}$, Maxim Ziatdinov ${ }^{2}$, Xiaohang Zhang ${ }^{3}$, Rama Vasudevan ${ }^{4}$, Eugen Eliseev ${ }^{5}$, Anna Morozovska $^{6}$, Ichiro Takeuchi ${ }^{3}$ and Sergei Kalinin ${ }^{7}$

${ }^{1}$ Oak Ridge National Laboratory, Oak Ridge, Tennessee, United States, ${ }^{2}$ Computational Sciences and Engineering Division, Oak Ridge National Laboratory, Oak Ridge, Tennessee, United States, ${ }^{3}$ University of Maryland, Maryland, United States, ${ }^{4}$ Oak Ridge National Laboratory, United States, ${ }^{5}$ Institute for Problems of Materials Science, National Academy of Sciences of Ukraine, United States, ${ }^{6}$ Institute of Physics, National Academy of Sciences of Ukraine, United States, ${ }^{7}$ Center for Nanophase Materials Sciences, Oak Ridge National Laboratory, Oak Ridge, Tennessee, United States

(Scanning) Transmission Electron Microscopy offers a plethora of detection channels and operating modes to provide diverse, chemical, structural, electrical, etc, information down to atomistic scales. Generally, the unrealized ideal outcome in analyzing these multi-channel datasets is determination of the causal physics relationships connecting parameters, e.g. a change in chemistry driving a phase transition. But lacking access to individual independent parameters to ascertain causal directions (S)TEM analysis instead is usually limited to identifying correlative relationships as in dimensionality reduction methods. This is an especially important factor for the surge in machine learning applications which are correlative in nature and subject to confounding factors (moreover the links are generally obfuscated in complexity). In this work we implement a workflow for causal analysis of HR-STEM data in a complex oxide thin film system possessing multiple competing degrees of freedom with proxies in the atomic fine-structure of HAADF images. We subject derived STEM parameters to information-geometric causal inference (IGCI) and additive noise models (ANM) to establish the pairwise causal directions [1]. We also employ a linear non-gaussian acyclic model (LiNGAM) [2] to reconstruct the causal chain and estimates of linear connection coefficients between the observed variables.

The model system employed was a doped ferroelectric, $\mathrm{Sm}_{\mathrm{x}} \mathrm{Bi}_{1-\mathrm{x}} \mathrm{FeO}_{3}$, analyzed across multiple compositions from a single combinatorial thin film growth. These $\mathrm{ABO}_{3}$ perovskites exhibit numerous symmetry lowering degrees of freedom from the cubic host phase, in this case a rhombohedral ferroelectric phase for at low $x$, and an antiferrodistortive orthorhombic phase for large $x$, with a wide region of phase coexistence and border phases [2]. Descriptors were defined from local (unit-cell) atomic HAADF STEM (Fig 1), corresponding to structural (a,b,a/b,ab $\Delta, \theta, \mathrm{Vol})$, compositional (I1-I5), and polarization (P) parameters. The causal chain of these parameters was determined in pairwise fashion using IGCI and ANM across $\mathrm{x}=0-20 \%$ Sm compositions (example for $\mathrm{x}=0$ is shown in Fig $2 \mathrm{a}, \mathrm{b}, \mathrm{d}, \mathrm{e}$ ). Likewise the causal chain and linear coefficients were determined via LiNGAM across the same compositions ( $\mathrm{x}=0$ in Fig 2c,f). Generally, chemical effects including local composition and molar volume are found to be higher on the causal chain, polarization effects being secondary, and tetragonality and differential chemical contrast the weakest. 
(a)
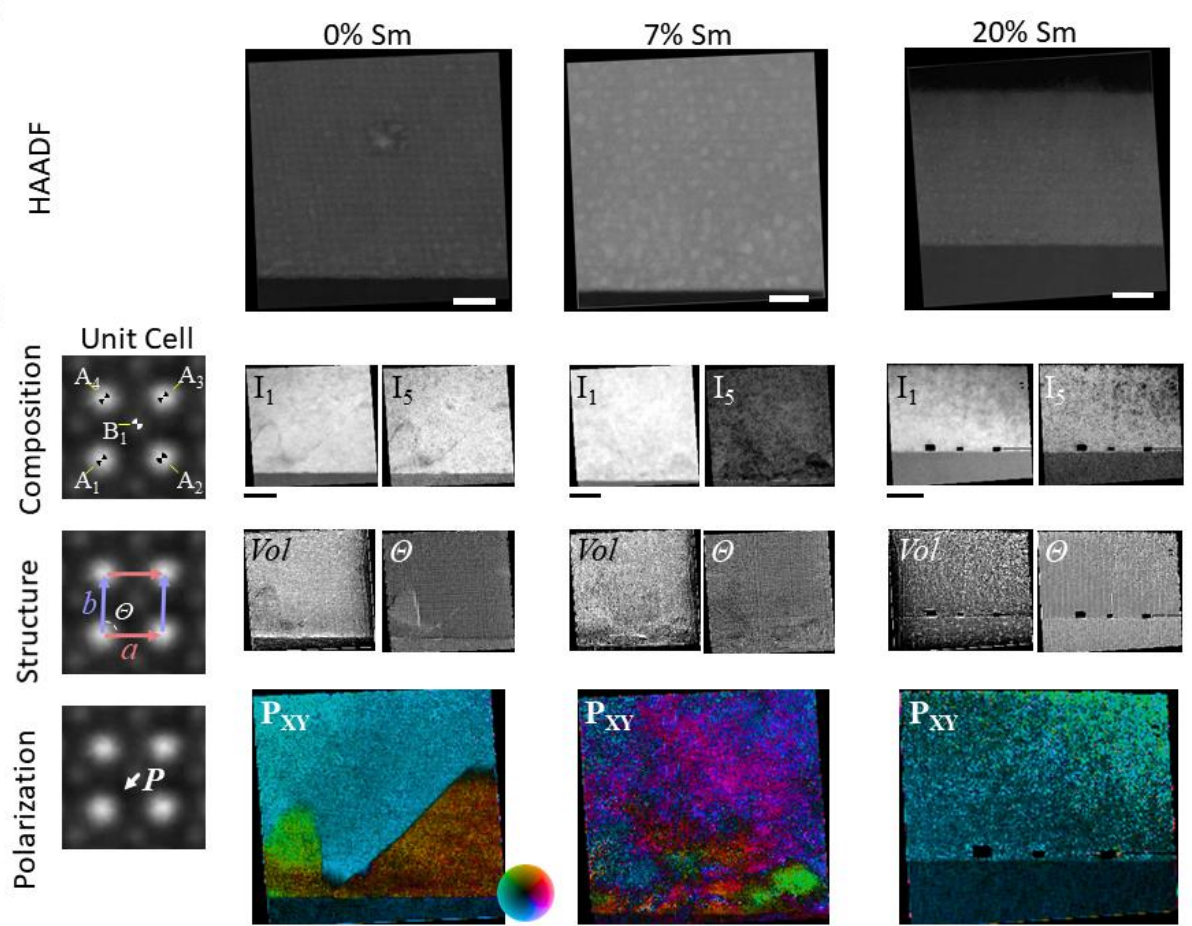

Figure 1. Figure 1. (a) HAADF sectional images from select compositions of a combinatorial a SmxBi1xFEO3 thin film. 10nm scalebars. (b) Selected B-site centered ABO3 unit-cell descriptors linked to composition, structure, an electrical polarization parameters of the host $\mathrm{Sm:BiFeO} 3$ system. Images are shown mapped to the a,b lattice space. 40 unit cell scalebars

(a)

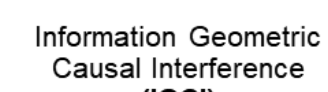
(IGCI)

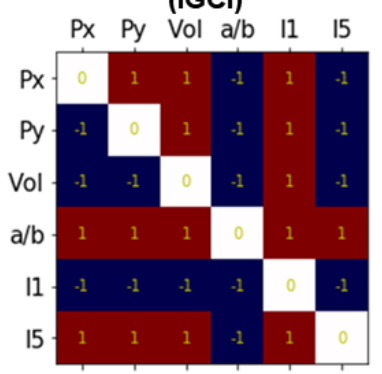

(d)

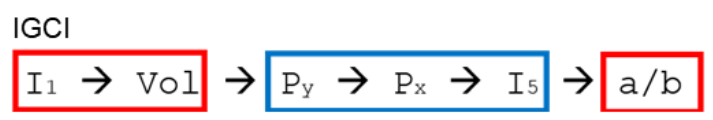

(e)

$$
\begin{aligned}
& \text { ANM } \\
& \mathrm{P}_{\mathrm{y}} \rightarrow\left(\mathrm{P}_{\mathrm{x}}, \mathrm{a} / \mathrm{b}, \mathrm{I}_{1}\right) \rightarrow \mathrm{Vol} \rightarrow \mathrm{I}_{5}
\end{aligned}
$$

(c)

Linear Non-Gaussian Acyclic Model (LiNGAM)

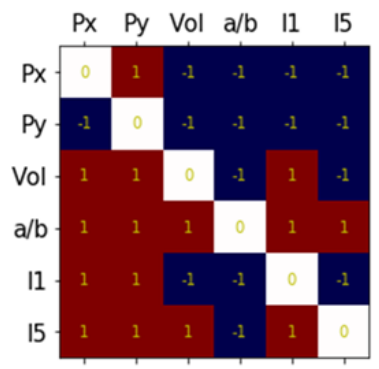

(f)
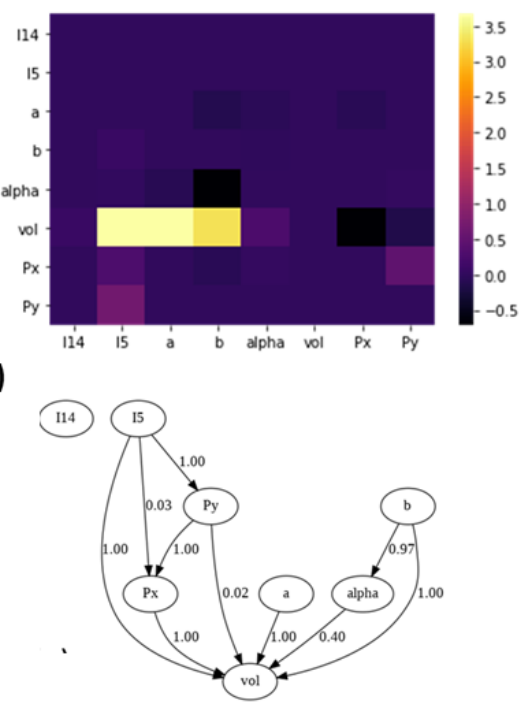
Figure 2. Figure 2. Causal matrices and chains for $\mathrm{x}=0$. (a) Causal sieve matrix for IGCI and (b) ANM. (c) Adjacency matrix for LiNGAM. Causal Chains for (a) IGCI, (b) ANM, and (c) LiNGAM.

References

[1] P. Daniusis et al., Proceedings of the 26th Conference on Uncertainty in Artificial Intelligence (UAI) 7 (2010), 1.

[2] S. Shimizu et al., Mach. Learn. Res. 7 (2006), p. 2003

[3] This work was supported by the U.S Department of Energy, Office of Science, Basic Energy Sciences, Materials Sciences and Engineering Division. 